\title{
Design of a Tele-operable Soft Actuator
}

\section{P.D.S.H. Gunawardane, R.E.A. Pallewela and Nimali T. Medagedara}

\begin{abstract}
Communication delay between robotic control systems and the robotic actuator is a major issue since the soft robotic motion requires controls to be precise and have quick responses. This is also essential for real time control of robots where the user should be able to send and receive signals with minimum delay to emulate a real time control. Specifically, in soft robotics, the communication delay could become a bigger issue due to the fact that soft robots require mode of pressure exerted into the actuator to make any kind of motion. A delay in such instances could cause the whole system to malfunction and even could cause the actuator to explode in the case of rapid and subtle changes in internally applied pressure. In this research, available IoT (Internet of Things) related systems and models have been studied and suitable IoT system was designed. The main focus of this research was to study the communication delay in IoT embedded hand gesture controlled soft robot finger (actuator). Two separate data processing units have been introduced in the master side and in the slave side to rapid capturing and sending data. By using this approach, processing delay is expected to be minimized. Transmission delay, propagation delay and queuing delay are found to be handled through external factors. These factors could be changed by using various kinds of servers and service providers. Soft elastomer actuator was designed and developed using standard rapid prototyping and soft lithography techniques generally used in soft robotics. Testing, data collecting and analyzing the system are carried out at various locations in Sri lanka and finally the Round Trip Delay Time (RTD) for each case was compared.
\end{abstract}

Keywords: $\quad$ IoT, Hand Gesture, RTD Delay Time, Soft Robot, Soft Actuator, Data Glove, Finite Element Method

\section{Introduction}

Soft Robotics is a sub field of robotics dealing with non-stiff robots constructed with soft and deformable material like silicon and rubber. It consists of low cost simple components and it can achieve complex motions. It allows for increased flexibility and adaptability to accomplish tasks, as well as provides improved safety when working around humans. These characteristics allow for its potential use in the fields of medicine and manufacturing.

Internet of things (IoT) [1] is a system of interrelated computing devices, mechanical and digital machines, objects or people, provided with unique identifiers and the ability to transfer data over a network without requiring human to human or human to computer interaction. Figure 1 shows the interconnected commonly used sensors and devices. IoT plays a major role in all aspects in the world. It covers various fields, including medical industry, automobile industry, mechanical devices, sports and homes etc.
IoT facilitates the way individuals communicate with nature and surroundings, and expands our social relationships with other people and IoT is

Eng. P.D.S.H. Gunawardane, AMIE(SL), MIEEE, B.Tech.Eng.(Hons) (OUSL), M.A.Sc. (UBC), PhD (Reading) $(U B C)$, Graduate Research Student in Department of Mechanical Engineering, The University of British Columbia, Vancouver, Canada.

Email:hiroshan@mail.ubc.ca

(iD) https://orcid.org/0000-0001-9195-6048

Eng. R.E.A. Pallewela, AMIE(SL), B.Tech.Eng. (OUSL), Department of Mechanical Engineering, The Open University of Sri Lanka.

Email:eashanmax@gmail.com

Eng. (Mrs) T.M.D.N.T. Medagedara, C.Eng., MIE(SL), MIEEE, B.Sc. Eng.(Hons) (Peradeniya), MPhil. (SHU), Senior Lecturer in Department of Mechanical Engineering, The Open University of Sri Lanka.

Email:tmmed@ou.ac.lk

(iD https://orcid.org/0000-0002-7210-0874

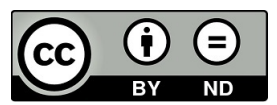

This article is published under the Creative Commons CC-BY-ND License (http:// creativecommons.org/licenses/by-nd/4.0/). This license permits use, distribution and reproduction, commercial and non-commercial, provided that the original work is properly cited and is not changed in anyway. 


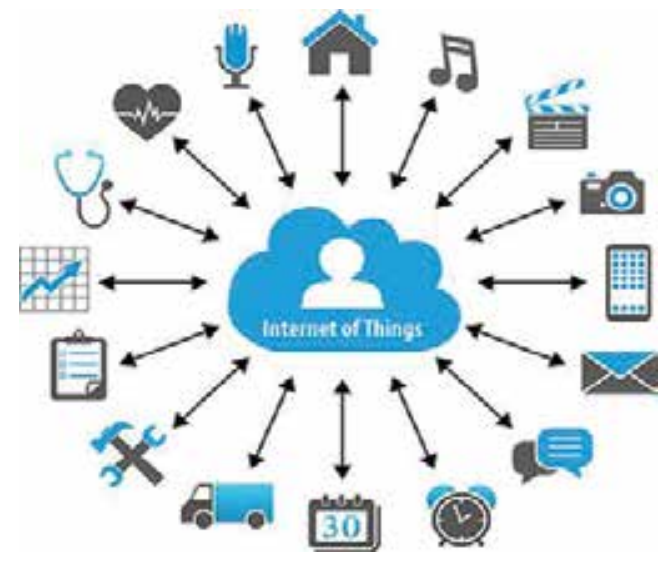

Figure 1 - Inter connected commonly used sensors and devices [2]

another revolutionary innovation that has provided immense benefits and influenced developments in how communication should be carried out. IoT is a platform that permits objects, including humans, to interact with each other over the internet so that they would be controlled, manipulated or simply share information [3]. A typical IoT system consists of 5 layers: (1) hardware/robotic things layer, (2) network layer, (3) internet layer, (4) infrastructure layer, and (5) application layer. Their descriptions are given in Table 1 [4]. This architecture is highly helpful for robotic manipulation.

Developing applications for IoT could be a challenging task due to (i) high complexity of distributed computing, (ii) lack of general guidelines or frameworks that handle low level communication and simplify high level implementation, (iii) multiple programming languages, and (iv) various communication protocols [5]. IoT characterizes the interconnection of different devices with universal accessibility and built-in intelligence. IoT has already modified the way we interact with devices and provided us with novel networking and socializing capabilities through intermediary devices [6]. Several research papers have compared the operating systems by their architecture, structure, operation and resource management but these comparisons are restricted to a specific domain $[7,8,9]$.
Table 1 - IoT layers and descriptions

\begin{tabular}{|l|l|}
\hline Layer & Description \\
\hline Hardware & $\begin{array}{l}\text { Contains objects such as sensors, } \\
\text { electronic robots that either collect } \\
\text { data or are manipulated. }\end{array}$ \\
\hline Network & $\begin{array}{l}\text { Network connection allows } \\
\text { connection between devices using } \\
\text { modes such as long range 4G, 3G, } \\
\text { short range Wifi, Bluetooth, Near } \\
\text { Field Communication (NFC) and } \\
\text { medium long-range Microwave } \\
\text { Access (WiMAX) and zigbee. }\end{array}$ \\
\hline In) & $\begin{array}{l}\text { This layer is used to send and } \\
\text { receive packet data, messages \& } \\
\text { TCP using protocols such as } \\
\text { MQTT, IPv6, DDS, AMQP, LLAP } \\
\text { \&CoAP. }\end{array}$ \\
\hline $\begin{array}{l}\text { Infrastru } \\
\text { cture }\end{array}$ & $\begin{array}{l}\text { This layer is the operating } \\
\text { system/platform for the IoT } \\
\text { system which would include } \\
\text { cloud based and standard OS. } \\
\text { Examples are Robotic Operating } \\
\text { System (ROS) Robot Service } \\
\text { Network Protocol (RSNP) \&RT } \\
\text { (Robot Technology) middleware. }\end{array}$ \\
\hline Application & $\begin{array}{l}\text { This is the end user GUI system } \\
\text { that is used to manipulate the } \\
\text { robotic system. }\end{array}$ \\
\hline
\end{tabular}

To enable such interactions, several competitive IoT application-layer communication protocols (e.g., extensible Messaging and Presence Protocol (XMPP) [10,11], HTTP REST [12-14], MQTT [15,16], and CoAP [17]) have been developed to satisfy the properties of constrained ecosystems such as IoT. Each protocol is designed for a particular set of application requirements and aspects of IoT communications, and these communication protocols blur the line between messaging mechanisms and semantic-based computation [18]. 


\section{$2 \quad$ Methodology}

\subsection{Experimental Detection of Round Trip Delay Time (RTD)}

This research mainly focuses on the development of a communication platform for a soft robotic finger which is controlled through a gesture controlled data glove [18], to study the reduction in the communication delays between two nodes, and suggest improvements for rapid response of the system. Current IoT system for soft robotics or solid actuator mainly faces data losses during data packet transferring in the communication system. Data loss during transmission between receiver and transmitter can occur due to interconnected breakdowns. Communication delays occur as a result of data losses in between two nodes. A designed system isneeded to be capable of controlling and monitoring from a long distance through the internet. The results of internet-enabled gesture controlled soft robot actuator provide a new level of flexibility, performance and cost advantages. Prevailing soft robot control systems are limited to local area networks or wire connected distance. On the performance of this developed system it is possible to communicate with actuator control station anywhere in the world (Figure 2).

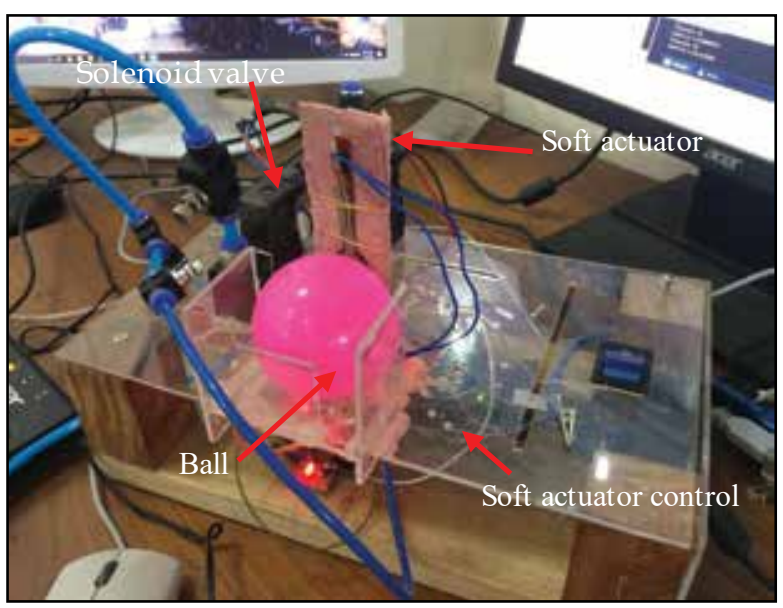

Figure 2 - Soft Finger control unit

The system uses a fabric based data glove as the input for the system (Figure 3). The data glove gives orientation data (index finger gesture angles) and force data which would then be processed using a Raspberry Pi 3, which in turn signals the output device via a communication platform which can be used as a global communication system. This data was used to control an output solenoid as shown in Figure 2, which used air pressure to bend the soft robotic actuator [22]. The server data was executed according to the program in actuator control board. Solenoid valve was used to control the actuator. A well-positioned camera, close to the soft robotic actuator, provided the feedback to the person who wears the data glove so as to adjust the motion accordingly (Figure 4).

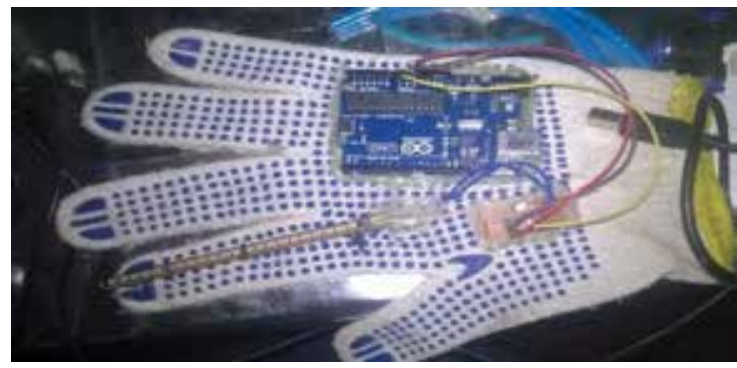

Figure 3 - Fabric based data glove

Data glove (Figure 3) was designed mainly for extracting parameters to detect the angle of bending and force on the ball. This unit consists of two voltage dividers and an analog to digital conversion unit.

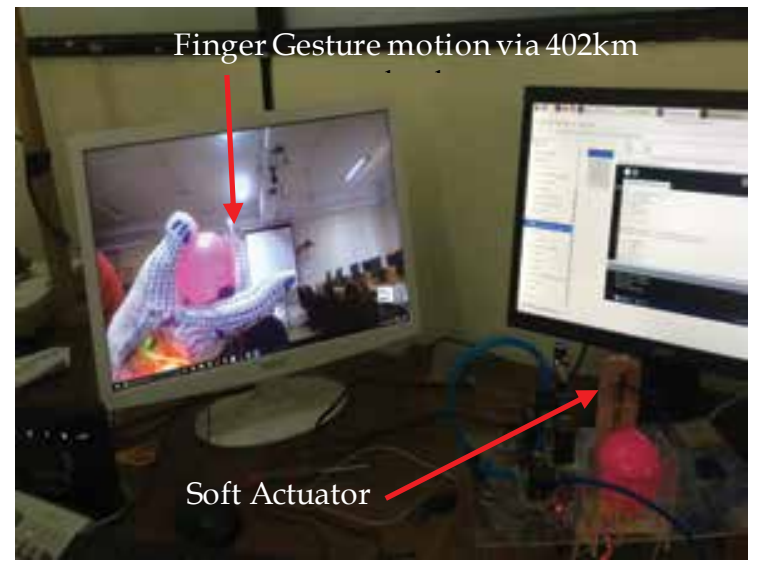

Figure 4 - Experimental setup for DG and soft actuator

MQTT Protocol (Figure 5) was used for the research as the communication platform as MQTT has ability to achieve better characteristics as a protocol to data transmission $[17,18]$. 


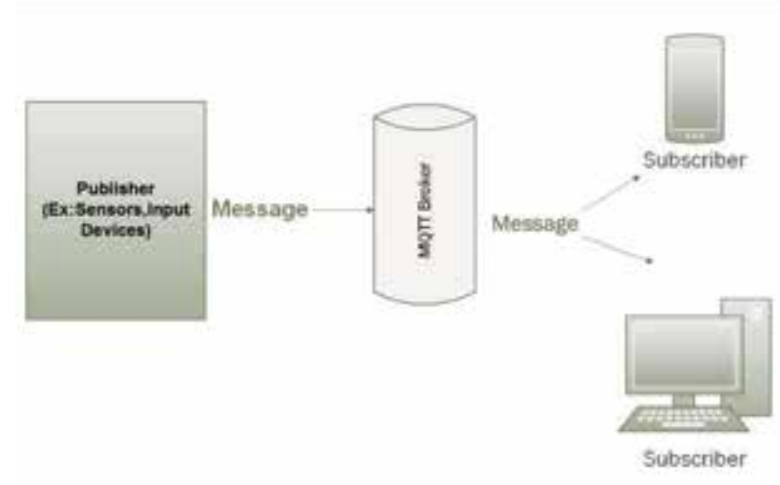

Figure 5 - MQTT broker diagram

The algorithm measured the Round Trip Delay Time (RTD) of the communication endpoints. RTD is the length of time taken to send signal and the length of time taken for an acknowledgment of the signal received. Delays between two network nodes are often asymmetric, and also the forward and reverse delays are not equal. Half the RTD value is the average of the forward and reverse delays, and therefore may be used sometimes as an approximation to the one-way delay (OWD).

\section{$R T$ Delay Time $=$ Average of Forward delays + Average of Reverse delays}

These delays were measured and the actuator was controlled by selecting the server properties (Table 2).

Table 2 - Server properties

\begin{tabular}{|l|l|}
\hline $\begin{array}{l}\text { Sri Lanka Service } \\
\text { Provider of server } \\
\text { IP }\end{array}$ & WSO2Pvt Ltd \\
\hline $\begin{array}{l}\text { Main Server } \\
\text { Location }\end{array}$ & $\begin{array}{l}\text { Singapore, 8071 km ahead } \\
\text { from Colombo }\end{array}$ \\
\hline Server IP & admin@18.216.10.83:1883 \\
\hline Server Model & Apache MQ \\
\hline Protocol & MQTT \\
\hline
\end{tabular}

\subsection{Design, 3D Printing \& Fabrication of Soft Bending Actuator}

Soft robot actuator design was verified and CAD models for the molds were created and 3D printed using Acrylonitrile Butadiene Styrene (ABS) at $200^{\circ} \mathrm{C}$ extruder temperature and the bed at $60^{\circ} \mathrm{C}$. These molds were used to fabricate the soft actuator [21].

\subsection{Finite Element Modelling and Analysis}

The behavior of the soft actuator was studied by Finite Element Analysis (FEA). Standard Abacus software was used to model the actuator and 8873 Tetrahedral Quadric Quadratic elements with four nodes were used (Figure 6) for the finite element analysis. Tetrahedral Quadratic elements are formulated in three-dimensional space with three degrees of freedom per node. These are the translational degrees of freedom in the $\mathrm{X}, \mathrm{Y}$ and $\mathrm{Z}$ directions.

The finite element model was used to find the required pressure for bending and study the behavior of the soft actuator, and also validate the physical experimental data. Behavior of the soft actuator was tested for the exact diameter used in the experiment (Figure 6).
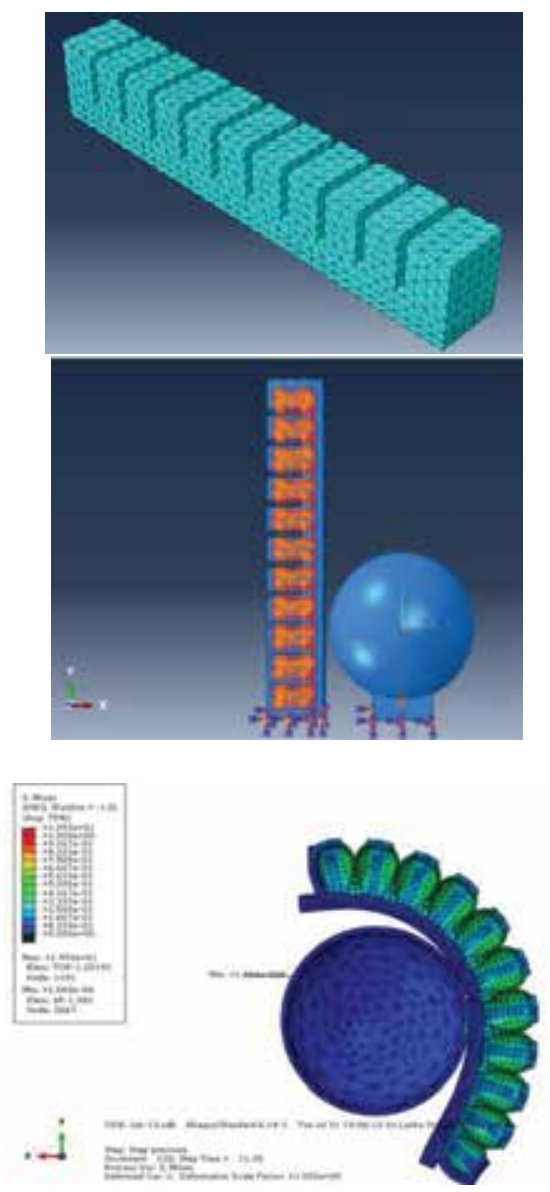

Figure 6 - Finite Element Analysis

Applying material data similar to elastomer materials with engineering properties, mesh, boundary conditions and loads, the behavior of 
the actuator was tested, and the results are shown in Figures 6 and 7.

\section{Results \& Analysis}

\subsection{FE Analysis}

Finite element method was applied to find out the required pressure for maximum bending of the soft actuator.

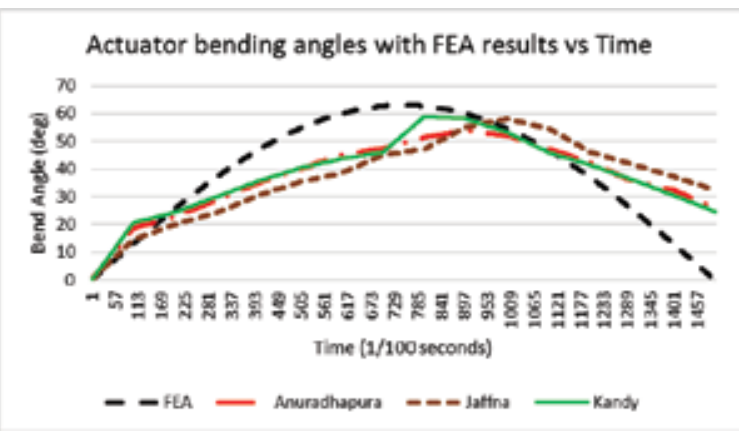

Figure 7 - Graph of angle of bending vs time

Without external loads and other factors, the soft actuator could reach the full bending at $60^{\circ}$ (to touch the ball) with $70 \mathrm{kPa}$ pressure without any failure of the material and the design. Figure 7 shows the experimental results of the bending behavior of the soft actuator in Jaffna, Anuradhapura and Kandy with the simulation results.

\subsection{Experimental Analysis}

RTD values for the corresponding bending angles were taken over $402 \mathrm{~km}$ (distance between Colombo and Jaffna), $200 \mathrm{~km}$ (distance between Colombo and Anuradhapura) and $133 \mathrm{~km}$ (distance between Colombo and Kandy) and as shown in Figures 8(a), 8(b) and 8(c), respectively.

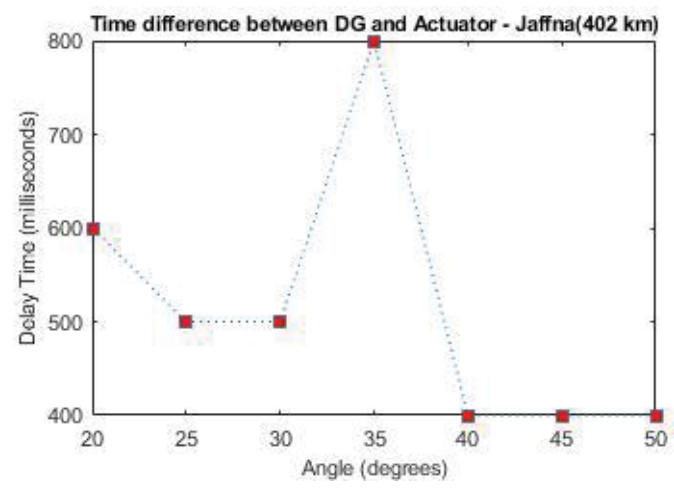

(a)

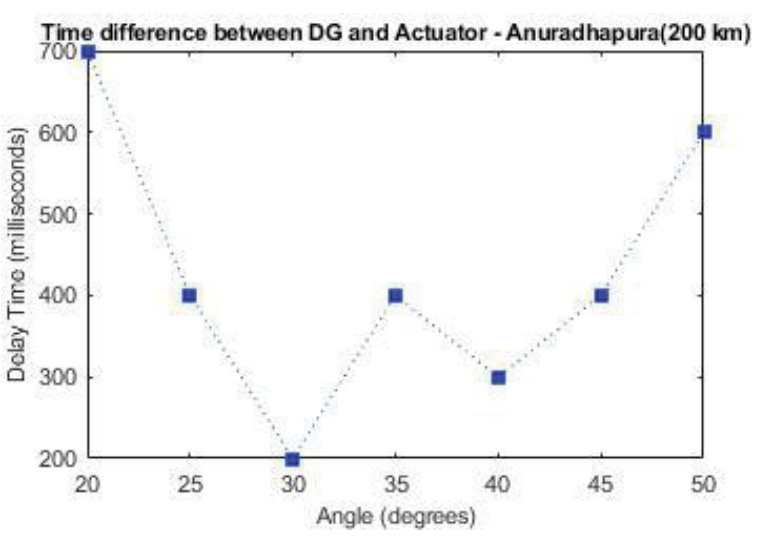

(b)

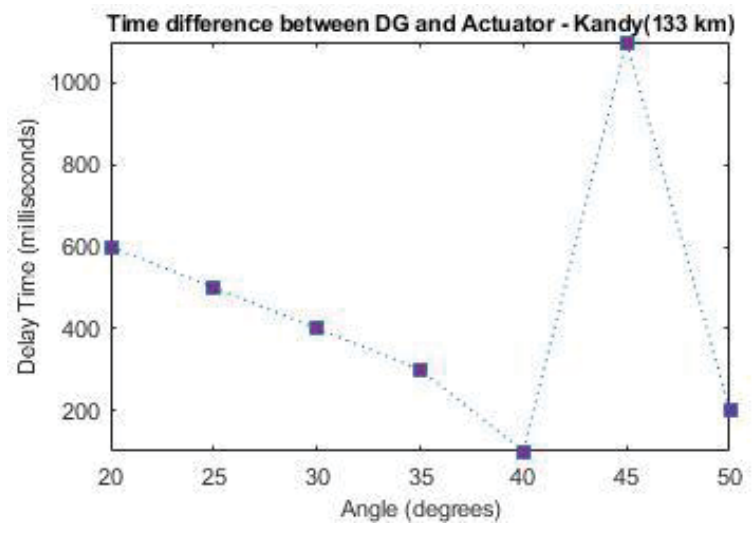

(c)

Figure 8 - Comparison of RTD values between DG and soft actuator

Table 3 - RTD values in different distances

\begin{tabular}{|c|c|}
\hline $\begin{array}{c}\text { Distance between } \\
\text { DG and Soft } \\
\text { actuator }(\mathrm{km})\end{array}$ & $\begin{array}{c}\text { Average } \\
\text { RTD }(\mathrm{ms})\end{array}$ \\
\hline 402 & 710.2 \\
\hline 200 & 688.23 \\
\hline 133 & 712.51 \\
\hline
\end{tabular}

\section{Conclusion}

The results show the comparison between the RTD values obtained from two remote areas Kandy $(133 \mathrm{~km})$ and Jaffna $(402 \mathrm{~km})$ according to the behavior of the soft actuator in the central point (Colombo). It shows some high delays from Kandy, which is suspected to be attributed to the mountainous terrain and the rainy climate present when the data was collected. Also, the reasons for these variations of the system could be due to the disturbances or the weakness of the strength of the communication signal, whereas 
the high delay in Jaffna could have been a consequence of insufficient $4 \mathrm{G}$ facilities.

In a good communication network, the value of the end to end delay is half of the RTD value. However, the quality of service also depends on the service provider and other aspects.

Finite element method was used to study the behavior of the soft bending actuator and the results show good agreement with the experimental behavior of the soft finger fabricated by elastomer rubber material.

\section{Acknowledgments}

Authors would like to thank the Engineering Trainees APDT Pathirana and Pradeep Rathnayake for their valuable assistance in this project, and would also like to express their gratitude to WSO2 Pvt. Ltd for providing WSO2 server and its Senior Software Engineer Charitha Gunathilaka and his expertise whenever needed. This work was funded by the University Research Grant of The Open University of Sri Lanka.

\section{References}

1. Enterprise Applications, software Development Solutions|NINtec. [Online]. Available: http://www.gatewaytechnolabs.co.uk/internetthings. [Accessed: 29-Jul-2017].

2. IoT Maniac, MACHINE LEARNING FOR INTERNET OF THINGS. 2018 [Online]. Available: http://iotmaniac.com/wpcontent/uploads/2016/09/1.jpg. [Accessed: 03Aug- 2018]

3. Ammar, M., Russello, G., and Crispo, B., "Internet of Things: A Survey on the Security of IoT Frameworks," Iournal of Information Security and Applications, vol.38, pp. 8-27, 2018.

4. Rav, P. P., "Internet of Robotic Things: Concept, Technologies, and Challenges," IEEE Access, vol. 4, pp. 9489-9500, 2016.

5. Zeinab, K. A. M., and Elmustafa, S. A. A., "Internet of Things Applications, Challenges and Related Future Technologies," World Scientific News. [Online].Available: http://psid.icm.edu.pl/psid/element/bwmeta1.e lement.psjd-86f8be9b-ee85-43ea-9a0971f79f74684b. [Accessed: 03-Feb-2018].
6. Baskiyar, S., and Natarajan, M., "A Survey of Contemporary Real-time Operating Systems," Informatica, pp. 233-240, 2005.

7. Grønli, T.-M., Hansen, I., and Ghinea, G., "Android vs Windows Mobile vs Java ME," Proceedings of the 3rd International Conference on PErvasive Technologies Related to Assistive Environments - PETRA 10, 2010.

8. Chandra, T. B., Verma, P., and Dwivedi, A. K., "Operating Systems for Internet of Things," Proceedings of the Second International Conference on Information and Communication Technology for Competitive Strategies - ICTCS 16, 2016.

9. Miorandi, S. Sicari, F. D. Pellegrini, and I. Chlamtac, "Internet of things: Vision, applications and research challenges," Ad Hoc Networks, vol. 10, no. 7, pp. 1497-1516, 2012.

10. Extensible Messaging and Presence Protocol (XMPP): Instant Messaging and Presence. [Online]. Available: https://xmpp.org/rfcs/rfc3921.html. [Accessed: 03-Feb-2018].

11. XMPP is the open standard for messaging and presence. [Online]. Available: https://xmpp.org/. [Accessed: 03-Feb-2018].

12. "Hypertext Transfer Protocol -- HTTP/1.1," IETF Tools. [Online]. Available: https://tools.ietf.org/html/rfc2616. [Accessed: 19-Oct-2017].

13. Feng, X., Shen, J., and Fan, Y. "REST: An Alternative to RPC for Web Services Architecture," 2009 First International Conference on Future Information Networks, 2009.

14. Karagiannis, V., Chatzimisios, P., VazquezGallego, F., and Alonso-Zarate, J., "A Survey on Application Layer Protocols for the Internet of Things," Transaction on IoT and Cloud Computing 2015, pp. 11-17, 2015.

15. MQ telemetry transport connectivity protocol. [Online].

Available:https://www.ibm.com/support/know ledgecenter/en/SSFKSJ_7.1.0/com.ibm.mq.doc/t t20045_htm. [Accessed: 19-Oct-2017].

16. MQ Telemetry Transport (MQTT) V3.1 Protocol Specification," IBM - United States, 19-Aug-2010. ¡Online]. Available: https://www.ibm.com/developerworks/library /ws-mqtt/. [Accessed: 19-Oct-2017]. 
17. "Publish-Subscribe Broker for the Constrained Application Protocol (CoAP)," IETF Tools. [Online]. Available: https://tools.ietf.org/html/draft-koster-corecoap-pubsub-00. [Accessed: 19-Oct-2017].

18. Sánchez, M. Batet, D. Isern, and A. Valls, "Ontology-based Semantic Similarity: A New Feature-Based Approach," Expert Systems with Applications, 20-Jan-2012. [Online]. Available: https://www.sciencedirect.com/science/article/ pii/S0957417412000954. 「Accessed: 12-Jan2020|A. E. Khaled and S. Helal, "Interoperable communication framework for bridging RESTful and topic-based communication in IoT," Future Generation Computer Systems, 2018.

19. Gunawardane, P. D. S. H. and Medagedara, N. T., "Comparison of Hand Gesture Inputs of Leap Motion Controller \& Data Glove in to a Soft Finger," 2017 IEEE International Symposium on Robotics and Intelligent Sensors (IRIS), 2017.

20. Abdou, A., Matrawy, A., and Oorschot, P. C. V., "Accurate One-Way Delav Estimation with Reduced Client Trustworthiness," IEEE Communications Letters, vol.19, no. 5, pp. 735-738, 2015.

21. "Modeling and Design Tool for Soft Pneumatic Actuators," Soft Robotics Toolkit. Online].Available: https://softroboticstoolkit.com/book/modelingsoft-pneumatic-actuators

22. Gunawardana, P.D.S.H., Pallewela, R.E.A., Nimali T. Medagedara, "KalmanFilter Based Approach to Overcome Elastic Saturation and Pneumatic Disturbances in Tele-operated Soft Actuators" Advances in Manufacturing Technology XXXIII IOS press 2019, doi:10.3233. 